Quaderni di Geografia Cahiers de Gécongaty rataphie

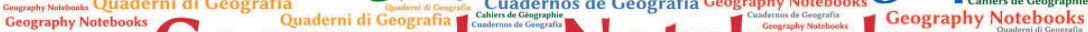
Cuadernos de Geografia Geography Notebooks $U 201000$ Cahiers de Géographi Cahiers de Géographie cahiers de Géographie Cuadernos de Geografía Cahiers de Géographie Cahiers de Géographie Cuadernos de Geografía Geography Notebooks

\author{
$4(2021)$ \\ 1 \\ Teatro di suoni. \\ Spazi acustici teatrali e territoriali
}

A cura di

Martino Mocchi, Lorena Rocca, Demis Quadri and Carlotta Sillano

EDITORIAL

Teatro di suoni per l'attaccamento ai luoghi. Uno sguardo geografico 11

Lorena Rocca

Per un teatro di suoni. Riflessioni su possibili dimensioni sonore nelle 23 creazioni site-specific di physical theatre

Demis Quadri

INTRODUCTION

Teatro di suoni. Spazi acustici teatrali e territoriali

Demis Quadri e Lorena Rocca

SPECIAL Issue

I suoni di Mantova come strumenti di interpretazione del paesaggio.

Tra turismo sostenibile ed educazione al patrimonio culturale

Valeria Pecorelli, Franca Zuccoli, Alessandra De Nicola, Enrico Squarcina

Il paesaggio sonoro campano tra contemporaneità e nuove forme

di progettualità turistica

Germana Citarella 
La narrazione spettacolarizzata del paesaggio sonoro.

Da Giuseppe Chiari a Philip K. Dick e oltre

Francesco Michi

Musica di paesaggi sonori. Enunciazione, risignificazione, comunicazione

Carlotta Sillano

Camminare per ascoltare. Partiture invisibili del territorio abitato

Elisabetta Senesi

Il paesaggio sonoro in relazione. Suono, movimento e immagini per stimolare complessità percettiva Angela Calia

Groove Fields. Understanding the Dance Floor from an Art-Based Research Perspective

Sebastian Mattbias

Il silenzio come esperienza trasformativa. L'importanza del silenzio nella meditazione e in ambito professionale

Sebastiano Caroni

Progettare il silenzio. Una lettura acustica dell'ex villaggio sanatoriale 125 Morelli a Sondalo

Martino Mocchi

Voicing One's Will. Theatre as Audio-Visual Hypotyposis of the Poetic

Michael Groneberg

Music and Clowning in Europe, 20th-21st centuries

Anna Stoll Knecht

Il paesaggio sonoro nella composizione musicale. Un percorso bibliografico

Stefano Alessandretti

\#exploreART: il labirinto di A. Pomodoro e i bambini. Un progetto di fruizione condivisa con percorsi sensoriali partecipati Alessandra De Nicola, Franca Zuccoli 


\section{OTHER EXPLORATIONS}

Il rumore lontano. Intervista a Lorena Rocca

a cura di Martino Mocchi

Re Cervo. Intervista a Antonella Astolfi

a cura di Krizia Bonaudo e Demis Quadri

Centovalli-Centoricordi. Intervista a Oliviero Giovannoni a cura di Krizia Bonaudo e Demis Quadri

Alla ricerca di un metodo: Open Space Technology 



\title{
La narrazione spettacolarizzata del paesaggio sonoro. Da Giuseppe Chiari a Philip K. Dick e oltre
}

Francesco Michi

Musicista, sound artist, Forum Klanglandschaft - FKL

DOI: https://doi.org/10.7358/gn-2021-001-mich

\begin{abstract}
Through the description of some works that I have conducted in recent years with Mechi Cena and Maurizio Montini, I will try to outline some possibilities in which the narration of the listening, of what you listen to, can become a matter of theater, meaning theater as a place where sounds, in this case, are presented in a certain way and in a certain way they are listened to. The theater is not only, we know that, a certain architectural space, but a place in which there is a here and a there, things represented and the target audience of those representations, and the representations are, in this case, stories of sounds.
\end{abstract}

Keywords: theater; soundscape; listening; narration; time.

Parole chiave: teatro; paesaggio sonoro; ascolto; narrazione; tempo.

\section{InTRODUZIONE}

Da qualche anno la ricerca che conduco, da solo e insieme a Mechi Cena e con Maurizio Montini (per la parte tecnica), si è concentrata sulla narrazione del suono, o meglio delle esperienze sonore e di ascolto. $\mathrm{Ne}$ conseguono lavori, progetti, ipotesi che in qualche modo mi sembrano 
avvicinarsi, nella forma che prendono nel mio immaginario, a un certo tipo di teatro, laddove però è il paesaggio sonoro stesso a essere il protagonista e non solo sottofondo o effetto sonoro ${ }^{1}$.

Sarebbe scorretto sostenere che nel teatro non siano esistite ed esistano operazioni che vedono il suono di per sé costituire, quasi sempre comunque insieme alla luce, l'intero impianto drammaturgico della scena: per restare in ambito italiano posso citare ad esempio esperimenti degli anni '80 del secolo scorso, come il lavoro di Marchingegno, di Cauteruccio e Pergola, o Iper-Urania di Simone Carella.

Se quello era un teatro nel quale i suoni "prendevano la scena", in questa particolare accezione di teatro dei suoni che ho in mente, il protagonista è il racconto del mondo sonoro percepito.

Vorrei dunque presentare tre lavori (Variazioni su Fuori, Orizzonti e I suoni non sono pietre), un paio già realizzati, l'altro in corso di realizzazione, scaturiti da questo approccio narrativo e che nel mio immaginario possono configurarsi come una forma di teatro sonoro.

\section{Variazioni su Fuori di Giuseppe Chiari}

Variazioni su fuori prende le mosse dal lavoro di Chiari ${ }^{2}$ intitolato, appunto, Fuori. La partitura di Fuori fu scritta da Chiari nel 1965, e pub-

${ }^{1} \mathrm{Ci}$ sarebbe peraltro da discutere sulla nuova consuetudine di chiamare "paesaggio sonoro" indicando l'insieme di suoni che popolano una rappresentazione teatrale, qualora non si tratti di colonna sonora/musica. Il termine "soundscape" tradotto con "paesaggio sonoro", così come è stato definito da Barry Truax nell'Handbook for Acostic Ecology (1978) e come anche molto più recentemente in Careggi Landscape Declaration on Soundscape (UNISCAPE 2012), fa riferimento all'attività di percezione del suono ambientale e non alla sua produzione. Il punto di vista per cui è lecito l'uso di "paesaggio sonoro" è dunque, a mio giudizio, radicalmente diverso. Forse, chissà, potrebbe essere più corretto usare qualcosa come "scenografia sonora", ampliando il concetto di scena a comprendere anche l'aspetto sonoro dell'ambiente progettato.

${ }^{2}$ Giuseppe Chiari (Firenze, 26/9/1926 - Firenze, 9/5/2007) è stato un pittore e compositore italiano. Fu musicista, pianista e artista visivo. Fece parte del movimento Fluxus. Inizia a scrivere musica dal 1950 [...]. Sue composizioni del primo periodo sono gli Intervalli e gli Studi sulla singola frequenza. Tra le opere successive Gesti sul piano del 1962 e L'arte è facile del 1972. All'interno del gruppo Fluxus sperimenta il concetto di "musica visiva" combinando l'arte visiva e musicale in un continuum dove la musica si caratterizza per la sua componente visiva. Sul fronte delle arti visive l'astrattismo e il concettuale conquistano irreversibilmente l'incorporeità tipica dell'espressione musicale (fonte: Wikipedia - https://it.wikipedia.org/wiki/Giuseppe_Chiari_compositore). 
blicata nel suo libro Musica senza contrappunto, fu composta per essere eseguita in situazioni da concerto, oppure negli happening che Fluxus organizzava. Fuori è una partitura verbale, non ci sono note, non ci sono indicazioni di durata, ma solo istruzioni.

In breve, le istruzioni indicano al performer come raccontare a un ipotetico pubblico ciò che sta ascoltando: dice come farlo e quando farlo, e come reagire a certi stimoli.

In un certo senso questi sono già i suoni del teatro: uno scricchiolio, un colpo di tosse, il rumore di fondo dell'illuminazione. Questi suoni non sono il contenuto sonoro del lavoro, ma sono l'oggetto di una interpretazione, che determina una narrazione. Narrato e narrando coesistono, distanziati dal tempo della percezione e dal rispetto delle regole della partitura.

Il nostro lavoro, le nostre variazioni sull'opera di Chiari, consistono nel trasportarne all'aperto l'esecuzione - una piazza, un giardino o altro - e nell'aumentare il numero degli esecutori. Nella versione pubblicata da ANTS Records gli esecutori sono stati otto, lo spazio un parco pubblico. In seguito ogni esecutore ha lavorato sulla sua esecuzione, l'ha trascritta e riscritta, eliminando incertezze e balbettamenti e cercando di costruire un suo stile, una sua linea narrativa. Abbiamo poi riportato il tutto in una sala per la restituzione. Il risultato appare essere una sorta di multi-racconto dello stesso paesaggio sonoro, dove i suoni sono stati sostituiti da una loro molteplice narrazione, gli esecutori disposti nello spazio della sala similarmente a come lo erano nel parco.

In questo caso il testo di Chiari non fa da mediazione fra i suoni del teatro e il pubblico, ma è un dispositivo per fare di un paesaggio sonoro l'oggetto di una operazione di narrazione e ascolto mediato ${ }^{3}$.

\section{I SUONI NON SONO PIETRE}

Nella sua unica conferenza il timido e al tempo stesso esaltato Philip Dick confessò che, allo stesso modo in cui molte persone asseriscono di avere memoria di loro vite passate, egli aveva memorie di altre sue vite presenti ${ }^{4}$.

\footnotetext{
${ }^{3}$ Maggiori particolari su questo lavoro su un mio articolo su Giornate sonore (Michi 2018) e nel cofanetto Variazioni su Fuori (Michi and Cena 2020). Inoltre: http://www. arteco.org/michi/htm/lavori/workshop_variazioni_su_fuori/pag.htm.

${ }^{4}$ https://www.youtube.com/watch?time_continue=201\&v=0LDv8fm_R7g\&featu- 
Su questa esperienza, della cui realtà Dick era sicuro, sono basati molti suoi racconti e libri. A ben pensarci poi, in una scala più ridotta, l'esperienza di Dick potrebbe essere una metafora di ciò che accade nella lettura ed anche nel teatro.

L'immersione totale nella lettura porta a "vivere", emozionalmente, una doppia vita. Immersi nella lettura i suoni e le immagini narrate appaiono ai nostri occhi e alle nostre orecchie, ce le immaginiamo, le costruiamo, per capire meglio ciò di cui stiamo leggendo... e qualora alziamo gli occhi dal libro, distogliendo da esso l'attenzione, suoni e immagini di un'altra vita, alla quale certo siamo più abituati, sostituiscono quella realtà. Due presenti uno sull'altro. Teatro e cinema sono due situazioni simili: Foucault (2010) li cita come esempio di luoghi in cui coesistono, sono simultaneamente e realmente presenti, più spazi fra loro incompatibili.

Se cinema e teatro sono dunque oggettivamente, o realmente, eterotopie, la lettura di un romanzo, lo sprofondare nel mondo dei suoni che leggiamo riguarda la capacità di immaginare suoni che non sentiamo, di ricostruirli, di crearci l'impressione di percezioni sonore: i due spazi incompatibili coesistono, ma nella nostra mente, nel nostro mondo percettivo "allargato".

Su questa capacità di immaginare suoni si basa l'audiodocumentario I suoni non sono pietre, quasi una cronaca commentata di una passeggiata sonora sui generis, nella quale un pubblico non occasionale, in qualche modo legato all'attività teatrale di Armunia al Castello Pasquini a Castiglioncello, è stato condotto ad esplorare acusticamente gli scavi, chiusi, del porto romano di San Giuliano, vicino a Rosignano, in provincia di Livorno. Un luogo particolare, immerso in quel silenzio che siamo soliti trovare negli scavi archeologici chiusi e non aperti a visitatori, ma allo stesso tempo isolato dal resto del mondo dal suono costante della fabbrica Solvay, che lavora giorno e notte.

Come abbordare una visita in uno spazio sonoro la cui attualità sonora è assolutamente di scarso interesse, se non applicando l'osservazione di Dick sulla presenza di più presenti? Come non applicarla chiedendo di attivare la nostra capacità di trasportarci in una sorta di ricostruzione percettiva immaginaria, che però rimane sovrapposta alla percezione attuale, con la quale si confronta e genera idee?

Richiamiamo sul palcoscenico delle nostre percezioni ciò che non

re=emb_logo [07/04/2020]. 
udiamo, ma siamo in grado di ricostruire con i mattoni che sono le nostre esperienze sonore, misceliamolo col nostro ascolto attuale.

L'audiodocumentario fu presentato come restituzione delle due passeggiate sonore condotte al festival Armunia nell'agosto $2017^{5}$.

\section{ORIZZONTE (O ORIZZONTI)}

Orizzonte, o Orizzonti, il titolo è ancora incerto, è il lavoro su cui sono attualmente occupato con Mechi Cena e Maurizio Montini. Ancora una coesistenza di mondi sonori. L'oggetto stavolta è il tempo, in se stesso certamente, ma anche come "portatore" del suono.

L'orizzonte non esiste come "oggetto reale", esiste solo nella nostra percezione: è quel posto fin dove il nostro senso (può essere la vista, l'udito, l'olfatto) riesce a percepire... Ma allo stesso tempo è una sorta di linea, che segnala un "di qua" e un "di là", ma anche un cambiamento di sostanza, acqua e aria, terra e aria: il sopra e il sotto che evidentemente appaiono (e sono) diversi.

La costruzione di Orizzonte (o Orizzonti) è costituita da un fronte di altoparlanti disposti davanti al pubblico come a formare una linea appena curva, come appunto la linea dell'orizzonte. Dalla nostra postazione, come pubblico, ascoltiamo il mondo appena al di là della linea del nostro orizzonte uditivo. Si è aperto uno squarcio, abbiamo la possibilità di ascoltare suoni da un Universo a noi parallelo (o se vogliamo ancora a un altro presente) del quale però la sostanza del tempo viene da noi percepita in modo diverso. E se il tempo funziona diversamente, il suono, che da questo è portato, che «sul tempo si adagia come un sultano sulla sua portantina» ${ }^{6}$, funziona in modo diverso.

Lo spazio, dunque è diviso in modo teatrale, di qua noi, di là loro. La linea dell'orizzonte come la linea del proscenio, al di là del quale le cose, la realtà è, o può essere, di una "sostanza" diversa.

Il tempo, al di là del nostro orizzonte, ci appare come saltellante, privo di una continuità, capace di anticipare, spezzettare e far regredire gli eventi. Non c'è da aspettarsi da un evento che accade di fronte a noi che questo possieda una sua continuità: può cominciare, interrompersi e riprendere più avanti, o annunciarsi prima ancora di compiersi. I suoni

\footnotetext{
${ }^{5}$ Per approfondimenti: http://www.arteco.org/michi/htm/lavori/i_suoni_non_ sono_pietre/pag.htm.

${ }^{6}$ Dal testo recitato nella installazione Orizzonti (Michi and Cena 2018).
} 
che lo descrivono ai nostri orecchi sono la fedele descrizione della nostra percezione di quell'universo.

Il tempo ancora, in un altro universo, si può per alcuni fermare, dando loro la possibilità di essere assaporato, così come avrebbe voluto Faust, che chiedeva solo di poter gustare, fermandolo, la bellezza di un attimo.

$\mathrm{E}$ ancora ascoltando un altro universo potrebbero apparirci reali quelle impressioni che abbiamo circa la velocità con cui scorre il tempo... per le quali talvolta alcuni minuti sembrano tanti di più oppure passare in un attimo, ma i nostri implacabili orologi ci garantiscono di un passare del tempo costante e ordinato. La nostra percezione di un altro universo potrebbe permetterci di provare in quello la realtà di questi individuali rallentamenti e accelerazioni.

Seduti di fronte all'orizzonte, come marinai che guardano la fine del mare e l'inizio del cielo, noi possiamo solo ascoltare, ascoltare attraverso un ipotetico squarcio, come un altro tempo, che percepiamo diverso dal nostro, trasporta e deforma gli elementi del paesaggio sonoro del "di là". Orizzonte, o Orizzonti (l'idea era di presentare quasi in simultaneità squarci di mondi diversi), è ancora in progettazione: una prima versione è stata presentata sotto forma di installazione al meeting di FKL - Forum Klanglandschaft a Vigevano nel 2018 (Michi and Cena 2018).

\section{Conclusioni?}

Non c'è, come suggerisce il titolo del paragrafo, una conclusione a questi racconti. L'ipotesi di una lettura di questi lavori che in qualche modo richiami una possibilità per un teatro di suoni è venuta a formarsi parallelamente e proprio a causa della stesura di questo intervento. Tutto è ancora in corso e prendere in considerazione anche questo punto di vista può aiutare una evoluzione, così come dirottare i canali di fruizione di questi stessi lavori.

Il teatro, d'altra parte, gioca con tre elementi principali: il luogo, la rappresentazione (termine che si identifica in molti casi proprio con quello di teatro), la fruizione o la percezione. Quest'ultimo elemento in questo contesto è particolarmente rilevante, perché la parola greca da cui

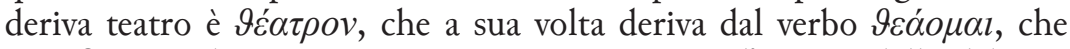
significa guardare, come a mettere in primo piano l'aspetto della elaborazione che lo spettatore fa dentro di sé di ciò a cui assiste. Per ogni spettatore tendenzialmente diversa. E così anche il paesaggio sonoro, nella interpretazione di Schafer e Truax è definito dalla presenza insostituibile di 
chi lo percepisce, e lo elabora. Scrive Albert Mayr: «in ogni caso, volendo essere rigorosi, il paesaggio sonoro lo "crea" l'ascoltatore nel momento in cui si appresta a percepire coscientemente un dato ambiente acustico; lo crea in base alle sue capacità percettive, le sue modalità appercettive del momento, al suo background culturale, a sue preferenze e/o idiosincrasie, e, molto probabilmente, al condizionamento da parte degli innumerevoli ambienti acustici virtuali che ci accompagnano quotidianamente. Dunque i paesaggi sonori di due soggetti che si trovano nello stesso momento nello stesso ambiente acustico possono essere significativamente diversi» $(2020,36)$.

Come dunque rendere possibile la fruizione teatrale di un reale paesaggio sonoro, che abbiamo visto definire come l'elaborazione di un soggetto percipiente, mantenendo una drammaturgia e la necessità di una "rappresentazione"? La narrazione potrebbe essere una risposta.

Quindi voglio concludere citando un importante lavoro di un musicista, che ancora può essere letto come modo di spettacolarizzare la narrazione del paesaggio, ma in modo ancora più immersivo.

In I'm Sitting in a Room di Alvin Lucier (1980), scritto nel 1969, narrazione, partitura, voce, luogo della performance - chiamiamolo teatro ${ }^{7}-$ effetto sonoro, il tempo necessario affinché tutto si realizzi sono realmente tutt'uno.

I'm Sitting in a Room è un brano parlato, il cui testo (che viene recitato) descrive quello che deve essere fatto per attivare un effetto sonoro che a sua volta attiva una ulteriore conoscenza delle capacità risonanti della stanza nella quale si è, così come delle forme più sottili della prosodia del narrato e della pronuncia.

Si è "attanti" e spettatori di una narrazione del suono della e nella stanza, che si dipana, come fosse una drammaturgia, fino ad arrivare alla sua essenza. Ogni particolare della nostra emissione sonora contribuisce al risultato, sempre diverso a seconda del luogo dell'azione. Lucier dice che questo brano è strettamente connesso col suo difetto di pronuncia, la sua balbuzie, che alla fine del processo messo in atto, non sarà più significante né, si suppone, imbarazzante. Ma c'è molto di più.

La versione di Lucier è stata pubblicata su cd da Lovely Music, si trova anche in mp3 e dura circa 45 minuti. Rimando per un ascolto a una versione realizzata da me molto più breve, in italiano, senza balbuzie

${ }^{7}$ Intendiamo per teatro (in questo caso per teatro di suoni) un luogo in cui i suoni vengono proposti e ascoltati in un 'certo modo', e sottolineo ascoltati, perché in fondo è questo quello che fa la differenza, e cioè la consapevolezza della loro non occasionalità. 
ma magari condita da un accento toscano, disponibile su Youtube (Michi 2008).

Il processo di Lucier, che in breve consiste nel registrare il testo narrato, diffondere nella stessa stanza e ancora registrare e poi diffondere finché sarà percepibile solo la frequenza di risonanza della stanza stessa, è stato anche da me più volte usato per produrre, in tempo reale, materiale sonoro estremamente site-specific per varie performance.

\section{RIFERIMENTI BIBLIOGRAFICI}

Chiari, G. 1969. Musica senza contrappunto. Roma: Lerici Editore.

Foucault, M. 2010. Eterotopie 1. Milano-Udine: Mimesis Edizioni.

Lucier, A. and S. Douglas. 1980. Chambers - Scores by Alvin Lucier. Middletown, Connecticut: Wesleyan University Press.

Mayr, A. 2020. "Ascolti mediati: aspetti del rapporto tra mondo della tecnica e paesaggio sonoro”. Musica/Tecnologia 14. Firenze: University Press e Fondazione Ezio Franceschini.

Michi, F. 2018. "Suonare l'ascolto. Le 'Variazioni su Fuori di Giuseppe Chiari”'. In Giornate sonore, a cura di Alessandra Calanchi e Massimiliano Morini. 65-79. Fano: Aras.

Michi, F. e M. Cena. 2020. Variazioni su Fuori. Roma: ANTS.

Uniscape. 2013. "Careggi Declaration on Soundscapes". Quaderni di Careggi, 3: 14-15.

Truax, B. 1978. The World Soundscape Project's Handbook for acoustic ecology. Vancouver, B.C.: A.R.C. Publications.

\section{RifERIMENTI Audiovisivi}

Michi, F. 2008. "I'm sitting in a room (Lucier)". https://www.youtube.com/watch?$\mathrm{v}=152 \mathrm{z} 0 \mathrm{~h} 7 \mathrm{~g} 7 \mathrm{o4}$. [07/04/2020].

Michi, F e M. Cena. 2018. Orizzonte. http://www.arteco.org/michi/htm/lavori/orizzonte/pag.htm. [07/04/2020]. 